\title{
Pacific
}

Journal of

Mathematics

\section{TAUT FOLIATIONS IN KNOT COMPLEMENTS}

TAO LI AND RACHEL ROBERTS

Volume 269 No. 1

May 2014 


\title{
TAUT FOLIATIONS IN KNOT COMPLEMENTS
}

\author{
TAO LI AND RACHEL ROBERTS
}

\begin{abstract}
We show that for any nontrivial knot in $S^{3}$, there is an open interval containing zero such that a Dehn surgery on any slope in this interval yields a 3-manifold with taut foliations. This generalizes a theorem of Gabai on zero frame surgery.
\end{abstract}

\section{Introduction}

A transversely orientable codimension-one foliation $\mathscr{F}$ of a 3-manifold $M$ is called taut [Gabai 1991] if every leaf of $\mathscr{F}$ intersects some closed transverse curve. The existence of a taut foliation in a 3-manifold $M$ provides much interesting topological information about both $M$ and objects embedded in $M$. If a closed 3-manifold $M$ contains a taut foliation, either $M$ is finitely covered by $S^{2} \times S^{1}$ or $M$ is irreducible [Novikov 1965; Reeb 1952; Rosenberg 1968]. If a closed 3-manifold $M$ contains a taut foliation, then its fundamental group is infinite [Haefliger 1962; Novikov 1965; Gabai and Oertel 1989] and acts nontrivially on interesting 1-dimensional objects (see, for example, [Thurston 1998; Calegari and Dunfield 2003; Palmeira 1978; Roberts et al. 2003]), and its universal cover is $\mathbb{R}^{3}$ [Palmeira 1978]. Taut foliations can be perturbed to interesting contact structures [Eliashberg and Thurston 1998; Kazez and Roberts 2014] and hence can be used to obtain Heegaard-Floer information [Ozsváth and Szabó 2004b]. In this paper we seek to add to the understanding of the existence of taut foliations by describing a new construction of taut foliations.

Let $k$ be a nontrivial knot in $S^{3}$. In his proof of the Property R conjecture, Gabai [1987b] showed that the knot exterior $M=S^{3} \backslash$ int $N(k)$ has a taut foliation whose restriction to the torus $\partial M$ is a collection of circles of slope 0 . Thus a zero frame Dehn surgery on $k$ yields a closed 3-manifold that admits a taut foliation obtained by adding disks along the boundary circles of the taut foliation of $M$. In this paper, we extend Gabai's theorem from zero frame surgery to any slope in an interval that contains 0 . Although we restrict attention to knots in $S^{3}$, the approach described in this paper applies more generally to manifolds $(M, \partial M)$ with boundary a nonempty

Partially supported by an NSF grant.

MSC2010: 57M25, 57M50, 57N10, 57R30.

Keywords: taut foliation, Dehn surgery, branched surface. 
union of tori and for which there exists a well-groomed sutured manifold hierarchy which meets each component of $\partial M$ only in essential simple closed curves.

Theorem 1.1. Let $k$ be a nontrivial knot in $S^{3}$. Then there is an interval $(-a, b)$, where $a>0$ and $b>0$, such that for any slope $s \in(-a, b)$, the knot exterior $M=S^{3} \backslash \operatorname{int}(N(k))$ has a taut foliation whose restriction to the torus $\partial M$ is a collection of circles of slope s. Moreover, by attaching disks along the boundary circles, the foliation can be extended to a taut foliation in $M(s)$, where $M(s)$ is the manifold obtained by performing Dehn surgery to $k$ with surgery slope $s$.

A group $G$ is called left-orderable if there is a total order on $G$ which is invariant under left multiplication. We thank Liam Watson for calling our attention to the following results.

Corollary 1.2. Let $k$ be a hyperbolic knot in $S^{3}$ and let $M(1 / n)$ denote the manifold obtained by $1 / n$ Dehn filling along $k$. Then there is some number $N=N(k)$ such that $\pi_{1}(M(1 / n))$ is left-orderable whenever $|n|>N$.

Proof. The surgered manifold $M(1 / n)$ is a homology $S^{3}$ and, by Thurston's hyperbolic Dehn surgery theorem [Thurston 1982], atoroidal when $|n|$ is sufficiently large (or, equivalently, when $1 / n$ is sufficiently small). Moreover, by Theorem 1.1, $M(1 / n)$ contains a transversely oriented taut foliation whenever $1 / n$ is sufficiently close to 0. It therefore follows from [Calegari and Dunfield 2003, Corollary 7.6] that $\pi_{1}(M(1 / n))$ is left-orderable.

Ozsváth and Szabó [2004c; 2004d] defined the Heegaard-Floer homology group $\widehat{H F}(Y)$ of a 3-manifold $Y$. In [Ozsváth and Szabó 2005], they define L-spaces as follows.

Definition 1.3 [Ozsváth and Szabó 2005, Definition 1.1]. A closed three-manifold is called an $L$-space if $H_{1}(Y ; \mathbb{Q})=0$ and $\widehat{H F}(Y)$ is a free abelian group of rank $\left|H_{1}(Y ; \mathbb{Z})\right|$.

L-spaces are therefore the closed 3-manifolds with the simplest possible Heegaard-Floer homology groups and the following is an important open question:

Question 1.4 [Ozsváth and Szabó 2004a, Question 11]. Is there a topological characterization of L-spaces (i.e., one that makes no reference to Floer homology)?

Ozsváth and Szabó proposed the following partial answer to this question:

Conjecture 1.5 [Hedden and Levine 2012, Conjecture 1]. If $Y$ is an irreducible homology sphere that is an L-space, then $Y$ is homeomorphic to either $S^{3}$ or the Poincaré homology sphere.

Approaches to understanding L-spaces have included investigations into the following two questions. Are L-spaces exactly those irreducible rational homology 
3-spheres which contain no transversely oriented taut foliation? Are L-spaces exactly those irreducible rational homology 3 -spheres which have non-left-orderable fundamental groups? (See [Boyer et al. 2012] for a nice survey.)

Conjecture 1.6 [Boyer et al. 2012, Conjecture 1]. An irreducible rational homology 3-sphere is an L-space if and only if its fundamental group is not left-orderable.

With Conjecture 1.6 in mind, we compare Corollary 1.2 with the following result, which appears in various contexts [Ozsváth and Szabó 2004b, Corollary 1.3; Ghiggini 2008, Corollary 1.5], but is stated most conveniently as [Hedden and Watson 2010, Proposition 5].

Proposition 1.7 [Ozsváth and Szabó 2004b; Hedden and Watson 2010]. Suppose k is a nontrivial knot in $S^{3}$ and let $M(1 / n)$ denote the manifold obtained by $1 / n$ Dehn filling along $k$. If $M(1 / n)$ is an L-space, then either $n=1$ and $k$ is the right-handed trefoil or $n=-1$ and $k$ is the left-handed trefoil.

It follows that Conjecture 1.5 holds for 3-manifolds obtained by surgery on knots in $S^{3}$. And it follows from Corollary 1.2 and Proposition 1.7 that Conjecture 1.6 holds for 3-manifolds obtained by $1 / n$ surgery on the complement of hyperbolic knots when $|n|$ is sufficiently large.

In Theorem 1.1, the interval $(-a, b)$ depends both on the knot $k$ and on the sutured manifold decomposition in [Gabai 1987b]. In [Roberts 2001a; 2001b], it is shown that if $k$ is a fibered hyperbolic knot (not necessarily in $S^{3}$ ), then this interval can always be chosen to contain $(-1, \infty),(-\infty, 1)$, or $(-\infty, \infty)$. Related results appear in [Dasbach and Li 2004; Delman and Roberts 1999; Roberts 1995]. Moreover, the values of $a$ and $b$ in a maximal such interval $(-a, b)$ reveal information about the pseudo-Anosov monodromy and hence the geometry of $M$.

Question 1.8. Let $k$ be a nontrivial knot in $S^{3}$, and let $a>0$ and $b>0$. What is the maximal interval $(-a, b)$ such that for any slope $s \in(-a, b)$, the knot exterior $M=S^{3} \backslash \operatorname{int}(N(k))$ has a taut foliation whose restriction to the torus $\partial M$ is a collection of circles of slope $s$, and the foliation can be extended to a taut foliation in $M(s)$ by attaching disks along the boundary circles, where $M(s)$ is the manifold obtained by performing Dehn surgery to $k$ with surgery slope $s$ ?

Conjecture 1.9. Such a maximal interval will always contain $(-1,1)$.

The proof of the main theorem uses theorems in [Li 2002; 2003] on branched surfaces to generalize the approach of [Roberts 2001a] to nonfibered knots. We first use Gabai's [1983; 1987a; 1987b] sutured manifold decomposition to construct a branched surface $B$. Then, after first splitting $B$ as necessary, we add in some product disks to get a new branched surface that carries more laminations which 
extend to taut foliations. The key point in the construction is to add branch sectors so that the new branched surface does not contain any sink disk. By [Li 2002; 2003], this means that the branched surface carries a lamination.

\section{Laminar branched surfaces}

Definition 2.1. A branched surface $B$ in $M$ is a union of finitely many compact smooth surfaces, glued together to form a compact subspace (of $M$ ) locally modeled on Figure 1, left (ignore the arrows in the picture for now).

Given a branched surface $B$ embedded in a 3-manifold $M$, we denote by $N(B)$ a regular neighborhood of $B$, as shown in Figure 1, right. One can regard $N(B)$ as an interval bundle over $B$. We denote by $\pi: N(B) \rightarrow B$ the projection that collapses every interval fiber to a point. As shown in Figure 1, right, the boundary of $N(B)$ consists of two parts: the horizontal boundary $\partial_{h} N(B)$ which is transverse to the $I$-fibers of $N(B)$, and the vertical boundary $\partial_{v} N(B)$ which is the union of subarcs of the $I$-fibers. The branch locus of $B$ is $L=\{b \in B: b$ does not have a neighborhood in $B$ homeomorphic to $\mathbb{R}^{2}$ \}. We call the closure (under the path metric) of each component of $B \backslash L$ a branch sector of $B . L$ is a collection of smooth immersed curves in $B$. Let $Z$ be the union of double points of $L$. We associate with every component of $L \backslash Z$ a normal vector (in $B$ ) pointing in the direction of the cusp, as shown in Figure 1, left. We call it the branch direction of this arc. Let $D$ be a disk branch sector of $B$. We call $D$ a sink disk if the branch direction of every smooth arc in its boundary points into the disk and $D \cap \partial M=\varnothing$. We call $D$ a half sink disk if $\partial D \cap \partial M \neq \varnothing$ and the branch direction of each arc in $\partial D \backslash \partial M$ points into $D$. Note that $\partial D \cap \partial M$ might not be connected.

Laminar branched surfaces were introduced in [Li 2002] as a branched surface with the usual properties in [Gabai and Oertel 1989] plus a condition that there is no sink disk. The notion of laminar branched surface was slightly extended to

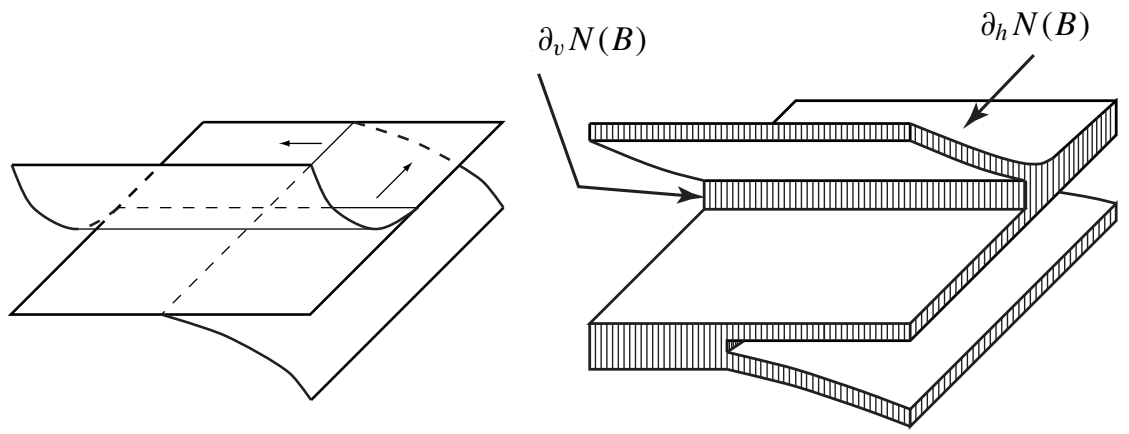

Figure 1. Left: a branched surface $B$. Right: a regular neighbor$\operatorname{hood} N(B)$. 
branched surfaces with boundary, by adding a requirement that there is no half sink disk [Li 2003]. Note that if a branched surface has no half sink disk, then one can arbitrarily split the branched surface near its boundary train track without creating any sink disk. This plus Theorem 1 of [Li 2002] implies the following theorem from [Li 2003]. Note that the condition that there is no sink disk basically guarantees that the branched surface carries a lamination and the other conditions in [Gabai and Oertel 1989] imply that the lamination is an essential lamination.

Theorem 2.2 [Li 2003, Theorem 2.2]. Let $M$ be an irreducible and orientable 3-manifold whose boundary is an incompressible torus. Suppose B is a laminar branched surface and $\partial M \backslash \partial B$ is a union of bigons. Then, for any rational slope $s \in \mathbb{Q} \cup \infty$ that can be realized by the train track $\partial B$, if $B$ does not carry a torus that bounds a solid torus in $M(s)$, then $B$ fully carries a lamination $\mathscr{L}$ whose boundary consists of loops of slope $s$ and $\mathscr{L}$ can be extended to an essential lamination in $M(s)$.

\section{Sutured manifold decompositions}

Gabai [1983] introduced the notions of sutured manifold and sutured manifold decomposition. We will state basic definitions and theorems as needed for this paper but we refer the reader to [Gabai 1983; 1987a; 1987b] for a more detailed description. The papers [Altman 2012; Cantwell and Conlon 2012; Juhász 2008] and book [Candel and Conlon 2003] also provide nice descriptions of some of Gabai's sutured manifold theory. In this paper, we will use branched surfaces to describe sutured manifolds and sutured manifold decompositions.

Definition 3.1 [Gabai 1983, Definition 2.6]. A sutured manifold $(M, \gamma)$ is a compact oriented 3-manifold $M$ together with a set $\gamma \subset \partial M$ of pairwise disjoint annuli $A(\gamma)$ and tori $T(\gamma)$. Furthermore, the interior of each component of $A(\gamma)$ contains a suture, that is, a homologically nontrivial oriented simple closed curve. We denote the set of sutures by $s(\gamma)$.

Finally, every component of $R(\gamma)=\partial M \backslash \operatorname{int}(\gamma)$ is oriented. Define $R_{+}(\gamma)$ (or $R_{-}(\gamma)$ ) to be those components of $\partial M \backslash \operatorname{int}(\gamma)$ whose normal vectors point out of (into) $M$. The orientations on $R(\gamma)$ must be coherent with respect to $s(\gamma)$; that is, if $\delta$ is a component of $\partial R(\gamma)$ and is given the boundary orientation, then $\delta$ must represent the same homology class in $H_{1}(\gamma)$ as some suture.

Roughly speaking, a sutured manifold is a 3-manifold together with extra information about $\partial M$. Given a sufficiently nice surface $S$ properly embedded in a sutured manifold $(M, \gamma)$, it is important to be able to cut $M$ open along $S$ while keeping track of corresponding boundary information. This is captured in the following definition. 
Definition 3.2 [Gabai 1983, Definition 3.1]. Let $(M, \gamma)$ be a sutured manifold and $S$ a properly embedded surface in $M$ such that every component $\lambda$ of $S \cap \gamma$ satisfies one of these three conditions:

(1) $\lambda$ is a properly embedded nonseparating arc in $\gamma$.

(2) $\lambda$ is a simple closed curve in an annular component $A$ of $\gamma$ in the same homology class as $A \cap s(\gamma)$.

(3) $\lambda$ is a homotopically nontrivial curve in a toral component $T$ of $\gamma$, and if $\delta$ is another component of $T \cap S$, then $\lambda$ and $\delta$ represent the same homology class in $H_{1}(T)$.

The surface $S$ defines a sutured manifold decomposition

$$
(M, \gamma) \stackrel{S}{\rightsquigarrow}\left(M^{\prime}, \gamma^{\prime}\right),
$$

where $M^{\prime}=M \backslash \operatorname{int}(N(S))$ and

$$
\begin{aligned}
\gamma^{\prime}= & \left(\gamma \cap M^{\prime}\right) \cup N\left(S_{+}^{\prime} \cap R_{-}(\gamma)\right) \cup N\left(S_{-}^{\prime} \cap R_{+}(\gamma)\right), \\
& R_{+}^{\prime}\left(\gamma^{\prime}\right)=\left(\left(R_{+}(\gamma) \cap M^{\prime}\right) \cup S_{+}^{\prime}\right) \backslash \operatorname{int}\left(\gamma^{\prime}\right), \\
& R_{-}^{\prime}\left(\gamma^{\prime}\right)=\left(\left(R_{-}(\gamma) \cap M^{\prime}\right) \cup S_{-}^{\prime}\right) \backslash \operatorname{int}\left(\gamma^{\prime}\right),
\end{aligned}
$$

where $S_{+}^{\prime}$ and $S_{-}^{\prime}$ are those components of $\partial N(S) \cap M^{\prime}$ whose normal vectors point out of and into $M^{\prime}$, respectively.

Definition 3.3 [Gabai 1987a, Definition 0.2]. A sutured manifold decomposition

$$
(M, \gamma) \stackrel{S}{\rightsquigarrow}\left(M^{\prime}, \gamma^{\prime}\right)
$$

is called well-groomed if for each component $V$ of $R(\gamma), S \cap V$ is a union of parallel, coherently oriented, nonseparating closed curves and arcs.

Definition 3.4 [Gabai 1987b, Definition 3.2]. Let

$$
(M, \partial M) \stackrel{S_{1}}{\rightsquigarrow}\left(M_{1}, \gamma_{1}\right) \stackrel{S_{2}}{\rightsquigarrow} \ldots \stackrel{S_{n}}{\rightsquigarrow}\left(M_{n}, \gamma_{n}\right)
$$

be a sequence of sutured manifold decompositions where $\partial M$ is a nonempty union of tori. Define $E_{0}=\partial M$. Define $E_{i}$ to be the union of those components of $E_{i-1} \backslash \operatorname{int}\left(N\left(S_{i}\right)\right)$ which are annuli and tori (i.e., if $M_{i}$ is viewed as a submanifold of $M$, then $E_{i}$ consists of those components of $\gamma_{i}$ which are contained in $\partial M$ ). The components of $E_{i}$ are called the boundary sutures of $\gamma_{i}$.

Definition 3.5. Let $(M, \gamma)$ and $(N, \tau)$ be sutured manifolds. We will call $(M, \gamma)$ a sutured submanifold of $(N, \tau)$, and write $(M, \gamma) \subset(N, \tau)$, if $M$ is a union of components of $N$ and $\gamma=\tau \cap M$.

If $(M, \gamma) \subset(N, \tau)$, then we write $(N, \tau) \backslash(M, \gamma)$ to denote the sutured manifold $(N \backslash M, \tau \backslash \gamma)$. 
Theorem 3.6 [Gabai 1987b, Lemmas 3.6 and 5.1]. Let $k$ be a knot in $S^{3}$. There is a well-groomed sutured manifold sequence

$$
(M, \gamma) \stackrel{S_{1}}{\rightsquigarrow}\left(M_{1}, \gamma_{1}\right) \stackrel{S_{2}}{\rightsquigarrow} \ldots \stackrel{S_{n}}{\rightsquigarrow}\left(M_{n}, \gamma_{n}\right)=(S \times I, \partial S \times I)
$$

of

$$
(M, \gamma)=\left(S^{3} \backslash \operatorname{int}(N(k)), \partial N(k)\right)
$$

such that $\partial S_{i} \cap \partial N(k)$ is a (possibly empty) union of circles for each $i, 1 \leq i \leq n, S_{1}$ is a minimal genus Seifert surface, and $S$ is a compact (not necessarily connected) oriented surface.

Sutured manifold decompositions determine branched surfaces. As described by Gabai in [1987b, Construction 4.6] (and detailed further in [Cantwell and Conlon 2012]), a sutured manifold decomposition sequence corresponds to building a (finite depth) branched surface, starting with $S_{1}$ and successively adding the $S_{i}$ 's. To see this, inductively construct a sequence of transversely oriented branched surfaces. Let $B_{1}=S_{1}$. So we may view $M_{1}$ as $M \backslash \operatorname{int}\left(N\left(B_{1}\right)\right)$, where $N\left(B_{1}\right)$ is a fibered neighborhood of $B_{1}$. As a sutured manifold $\left(M_{1}, \gamma_{1}\right)$, its suture $\gamma_{1}$ is the annulus $\overline{\partial M \backslash N\left(B_{1}\right)}$ and the two components of $\partial_{h} N\left(B_{1}\right)$ are the plus and minus boundaries $R_{+}\left(\gamma_{1}\right)$ and $R_{-}\left(\gamma_{1}\right)$ of the sutured manifold. We may view $R_{+}\left(\gamma_{1}\right)$ and $R_{-}\left(\gamma_{1}\right)$ as lying on the plus and minus sides of $S_{1}$ respectively and we assign a normal direction for $B_{1}=S_{1}$ pointing from the plus side to the minus side.

Suppose we have constructed a branched surface $B_{k}$ using the surfaces $S_{1}, \ldots, S_{k}$ in the sutured manifold decomposition, such that $M \backslash \operatorname{int}\left(N\left(B_{k}\right)\right)=M_{k}$ and the suture $\gamma_{k}$ of $\left(M_{k}, \gamma_{k}\right)$ consists of $\partial_{v} N\left(B_{k}\right)$ and a collection of annuli in the boundary torus $\partial M$. Now we consider the sutured manifold decomposition

$$
\left(M_{k}, \gamma_{k}\right) \stackrel{S_{k+1}}{\leadsto}\left(M_{k+1}, \gamma_{k+1}\right) .
$$

The surface $S_{k+1}$ has a normal vector. Then we can deform $B_{k} \cup S_{k+1}$ into a branched surface $B_{k+1}$ as follows:

(1) For each component of $\partial S_{k+1}$ that is not totally inside $\partial_{v} N\left(B_{k}\right)$, we can deform $B_{k} \cup S_{k+1}$ near $\partial S_{k+1}$ as in Figure 2, left, so that the normal directions of $B_{k}$ and $S_{k+1}$ are compatible in the newly constructed branched surface.

(2) For each component $c$ of $\partial S_{k+1}$ lying inside a suture $\partial_{v} N\left(B_{k}\right)$, we first slightly isotope $S_{k+1}$ by pushing $c$ into $R_{ \pm}\left(\gamma_{k}\right) \subset \partial_{h} N\left(B_{k}\right)$, then as shown in Figure 2, right, we can deform $B_{k} \cup S_{k+1}$ near $c$ into a branched surface. By the requirement of the normal directions in the sutured manifold decomposition, the normal directions of $B_{k}$ and $S_{k+1}$ are compatible in the newly constructed branched surface. 

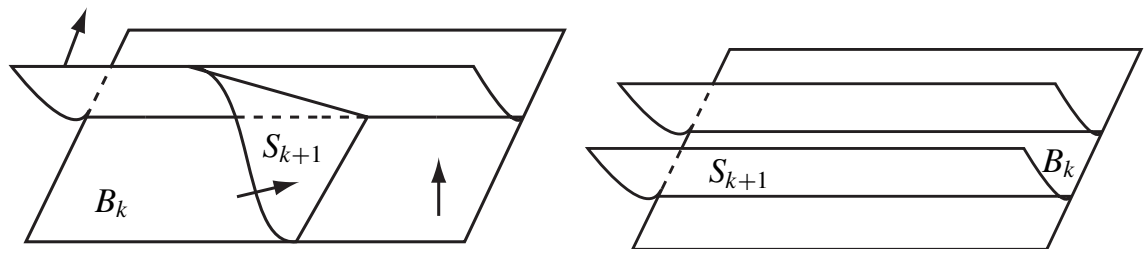

Figure 2. $B_{k} \cup S_{k+1}$ is deformed near $\partial S_{k+1}$. Left: the normal directions of $B_{k}$ and $S_{k+1}$ are compatible. Right: the neighborhoods of each suture are branched surfaces.

It follows from the definition of sutured manifold decomposition [Gabai 1983] that $M \backslash \operatorname{int}\left(N\left(B_{k+1}\right)\right)=M_{k+1}$ and the suture $\gamma_{k+1}$ of $\left(M_{k+1}, \gamma_{k+1}\right)$ consists of $\partial_{v} N\left(B_{k+1}\right)$ and a collection of annuli in the boundary torus $\partial M$. We will sometimes use the notation

$$
B_{k+1}=B_{\left(M_{k+1}, \gamma_{k+1}\right)}=B_{\left\langle S_{1} ; S_{2} ; \ldots ; S_{k+1}\right\rangle} .
$$

In summary, there is a map from the set of sutured manifold decomposition sequences to the set of properly embedded branched surfaces given by

$$
\left(S_{1}, S_{2}, \ldots, S_{l}\right) \mapsto B_{\left\langle S_{1} ; S_{2} ; \ldots ; S_{l}\right\rangle},
$$

and a (forgetful) map from the set of properly embedded branched surfaces to the set of sutured 3-manifolds given by

$$
B \mapsto\left(M_{B}, \gamma_{B}\right)=\left(M \backslash \operatorname{int}(N(B)), \partial_{v} N(B) \cup E^{\prime}\right),
$$

where $E^{\prime} \subset \partial M$ satisfies $E^{\prime}=E$, the set of boundary sutures, if $B$ intersects $\partial M$ only in longitudes. For future reference, it is useful to highlight that under this correspondence, $\partial_{h} N(B)$ corresponds naturally to $R_{+}\left(\gamma_{B}\right) \cup R_{-}\left(\gamma_{B}\right)$.

\section{The construction}

Modifying the sutured manifold hierarchy. Given a well-groomed sutured manifold hierarchy satisfying the conclusions of Theorem 3.6, we can inductively construct the sequence of branched surfaces $B_{1}, \ldots, B_{n}$ corresponding to the sutured manifold decomposition. The branched surface $B_{n}$ in the end has the properties that (1) $M \backslash \operatorname{int}\left(N\left(B_{n}\right)\right)$ is a product and (2) $\partial B_{n}$ is a collection of circles in $\partial M$ of slope 0 . In particular, any taut foliation carried by $B_{n}$ will also necessarily meet $\partial M$ only in simple closed curves of slope 0 .

To obtain a branched surface carrying taut foliations realizing an open interval of boundary slopes about 0 , it is necessary to modify the sutured manifold hierarchy, or, equivalently, the sequence of branched surfaces $B_{k}$. In this section, we describe one way of doing this. We break the process into two steps. 
As a first step, we slightly modify the sutured manifold hierarchy by adding some parallel copies of the surfaces $S_{k}$. Equivalently, we modify the sequence of branched surfaces $B_{k}$ by adding some parallel copies of the surfaces $S_{k}$. This operation is equivalent to a splitting of the branched surface. As a second (and final) step, we further modify the sutured manifold hierarchy by adding carefully chosen product disks.

Before giving a precise description of these steps, we introduce some terminology. Let $B$ be a transversely oriented branched surface and let $F$ be a component of $\partial_{h} N(B)$. The boundary of $F$ has two parts: $\partial F \cap \partial M$ and $\partial F \cap \partial_{v} N(B)$. We call $\partial F \cap \partial_{v} N(B)$ the internal boundary of $F$. Let $L$ be the branch locus of $B$. Let $L_{F}$ be the closure of $\pi^{-1}(L) \cap \operatorname{int}(F)$, where $\pi: N(B) \rightarrow B$ is the map collapsing each interval fiber to a point. So $L_{F}$ is a trivalent graph properly embedded in $F$. We call $L_{F}$ the projection of the branch locus to $F$. Each arc in $L_{F}$ has a normal direction induced from the branch direction of $L$.

Definition 4.1. Let $F$ be a component of $\partial_{h} N(B)$ with $\partial F \cap \partial M \neq \varnothing$ and let $\eta$ be an arc properly embedded in $F$. If $F$ has nonempty internal boundary, we require that $\eta$ connects $\partial F \cap \partial M$ to the internal boundary of $F$. Choose $\eta$ so that it intersects $L_{F}$ transversely and only at points in the interior of edges of $L_{F}$ (namely, it misses all triple points). Since $\eta$ is transverse to $L_{F}$, the induced branch direction of $L_{F}$ gives a direction along $\eta$ for each point in $\eta \cap L_{F}$. We say $\eta$ is $\operatorname{good}$ if these induced directions are coherent along $\eta$ and all point away from an endpoint of $\eta$ that lies in $\partial M$.

We say $F$ is good if $F$ satisfies the following properties:

(1) The closure of each component $D$ of $F \backslash L_{F}$ has a boundary arc with induced branch direction (from $L_{F}$ ) pointing out of $D$.

(2) If $F$ has internal boundary, then there is a set of disjoint good arcs, denoted by $\Gamma_{F}$, connecting each component of $\partial F \cap \partial M$ to the internal boundary of $F$.

(3) If $F$ has no internal boundary (in which case, $F$ must be a Seifert surface of the knot exterior), then there is a properly embedded nonseparating good arc in $F$, which we also denote by $\Gamma_{F}$.

Lemma 4.2. Let $B$ be a branched surface. If each component of $\partial_{h} N(B)$ is good, then $B$ does not contain any sink disk or half sink disk.

Proof. Let $F$ be a component of $\partial_{h} N(B)$ and let $L_{F}$ be as above. Let $P$ be the closure (under path metric) of a component of $F \backslash L_{F}$. So $P$ can be viewed as a copy of a branch sector of $B$. It follows from part (1) of Definition 4.1 that $B$ has no sink disk or half sink disk. 
Definition 4.3. We say the branched surface $B$ is good if

(1) every component of $\partial_{h} N(B)$ is good, and

(2) the arc systems $\Gamma_{F}$ as described in (2) and (3) in Definition 4.1 can be chosen so that the projections $\pi\left(\Gamma_{F}\right)$, as $F$ ranges over all components of $\partial_{h} N(B)$, are disjoint in $B$.

Note that these good $\operatorname{arcs} \Gamma_{F}$ will be the arcs along which we will attach product disks.

Step 1: Splitting $\boldsymbol{B}_{\boldsymbol{n}}$. Next we will describe the first modification of a sutured manifold decomposition sequence satisfying the conclusions of Theorem 3.6.

Lemma 4.4. Let $k$ be a nontrivial knot in $S^{3}$ and $M=S^{3} \backslash \operatorname{int}(N(k))$ the knot exterior. Let

$$
(M, \partial M) \stackrel{S_{1}}{\rightsquigarrow}\left(M_{1}, \gamma_{1}\right) \stackrel{S_{2}}{\rightsquigarrow} \ldots \stackrel{S_{n}}{\rightsquigarrow}\left(M_{n}, \gamma_{n}\right)=(S \times I, \partial S \times I)
$$

be a well-groomed sutured manifold hierarchy that satisfies the conclusions of Theorem 3.6. Then there exists a well-groomed sutured manifold hierarchy

$$
(M, \gamma) \stackrel{S_{1}}{\rightsquigarrow}\left(M_{1}^{\prime}, \gamma_{1}^{\prime}\right) \rightsquigarrow\left(M_{1}^{\prime \prime}, \gamma_{1}^{\prime \prime}\right) \stackrel{S_{2}}{\rightsquigarrow}\left(M_{2}^{\prime}, \gamma_{2}^{\prime}\right) \stackrel{R_{2}^{\prime}}{\rightsquigarrow}\left(M_{2}^{\prime \prime}, \gamma_{2}^{\prime \prime}\right) \stackrel{S_{3}}{\rightsquigarrow} \ldots \stackrel{S_{n}}{\rightsquigarrow}\left(M_{n}^{\prime}, \gamma_{n}^{\prime}\right)
$$

which also satisfies the conclusions of Theorem 3.6. Moreover, the branched surfaces $B_{l}^{\prime}=B_{\left(M_{l}^{\prime}, \gamma_{l}^{\prime}\right)}, 1 \leq l \leq n$, satisfy the conditions:

(1) $\partial B_{l}^{\prime} \cap \partial M$ is a collection of simple closed curves of slope 0 in $\partial M$ for each $l$.

(2) $\left(M_{l}, \gamma_{l}\right)$ is a sutured submanifold of $\left(M_{l}^{\prime}, \gamma_{l}^{\prime}\right)$ and $\left(M_{l}^{\prime}, \gamma_{l}^{\prime}\right) \backslash\left(M_{l}, \gamma_{l}\right)$ is a product sutured manifold for each $l$.

(3) Every branched surface $B_{l}^{\prime}$ is good.

(4) No B $B_{l}^{\prime}$ carries a torus.

(5) $\left(M_{n}^{\prime}, \gamma_{n}^{\prime}\right)$ is a product sutured manifold $\left(S^{\prime} \times I, \partial S^{\prime} \times I\right)$.

Proof. First note that, in the sutured manifold hierarchy above, each $R_{i}^{\prime}$ is a parallel copy of some components of $R_{+}\left(\gamma_{i}^{\prime}\right) \cup R_{-}\left(\gamma_{i}^{\prime}\right)$.

We proceed by induction on $l$. Since $k$ is nontrivial and hence $S_{1}$ has genus at least one, the branched surface $B_{1}^{\prime}=S_{1}$ is easily seen to satisfy conditions (1)-(4). So suppose we have constructed

$$
(M, \gamma) \stackrel{S_{1}}{\rightsquigarrow}\left(M_{1}^{\prime}, \gamma_{1}^{\prime}\right) \stackrel{R_{1}^{\prime}}{\rightsquigarrow}\left(M_{1}^{\prime \prime}, \gamma_{1}^{\prime \prime}\right) \stackrel{S_{2}}{\rightsquigarrow}\left(M_{2}^{\prime}, \gamma_{2}^{\prime}\right) \stackrel{R_{2}^{\prime}}{\rightsquigarrow}\left(M_{2}^{\prime \prime}, \gamma_{2}^{\prime \prime}\right) \stackrel{S_{3}}{\rightsquigarrow} \ldots \stackrel{S_{l}}{\rightsquigarrow}\left(M_{l}^{\prime}, \gamma_{l}^{\prime}\right)
$$

satisfying the conclusions of Theorem 3.6 and such that the corresponding branched surfaces $B_{i}^{\prime}=B_{\left(M_{i}^{\prime}, \gamma_{i}^{\prime}\right)}$ satisfy the conditions (1)-(4) for all $i, 1 \leq i \leq l$.

By condition (2), $\left(M_{l}, \gamma_{l}\right)$ is a sutured submanifold of $\left(M_{l}^{\prime}, \gamma_{l}^{\prime}\right)$. Let $R_{+}^{\prime}\left(\gamma_{l}\right)$ and $R_{-}^{\prime}\left(\gamma_{l}\right)$ be parallel copies of $R_{+}\left(\gamma_{l}\right)$ and $R_{-}\left(\gamma_{l}\right)$, chosen to be properly embedded in 
$\left(M_{l}, \gamma_{l}\right) \subset\left(M_{l}^{\prime}, \gamma_{l}^{\prime}\right)$ and with boundary lying in $E_{l} \cup A\left(\gamma_{l}\right)$ (see Definition 3.1 and Definition 3.4). Set $R_{l}^{\prime}=R_{+}^{\prime}\left(\gamma_{l}\right) \cup R_{-}^{\prime}\left(\gamma_{l}\right)$. We first consider the sutured manifold decomposition $\left(M_{l}^{\prime}, \gamma_{l}^{\prime}\right) \stackrel{R_{l}^{\prime}}{\rightsquigarrow}\left(M_{l}^{\prime \prime}, \gamma_{l}^{\prime \prime}\right)$. By the definition of $R_{l}^{\prime}$, this decomposition only adds some product complementary regions. Set $B_{l}^{\prime \prime}=B_{\left(M_{l}^{\prime \prime}, \gamma_{l}^{\prime \prime}\right)}$. The change from $B_{l}^{\prime}$ to $B_{l}^{\prime \prime}$ is basically the addition of branch sectors corresponding to $R_{l}^{\prime}$, and this operation creates some product complementary regions. See Figure 3, left, for a schematic picture. We may view $\left(M_{l}, \gamma_{l}\right)$ as a subset of $\left(M_{l}^{\prime \prime}, \gamma_{l}^{\prime \prime}\right)$, and consider the sutured manifold decompositions

$$
\left(M_{l}^{\prime}, \gamma_{l}^{\prime}\right) \stackrel{R_{l}^{\prime}}{\rightsquigarrow}\left(M_{l}^{\prime \prime}, \gamma_{l}^{\prime \prime}\right) \stackrel{S_{l+1}}{\rightsquigarrow}\left(M_{l+1}^{\prime}, \gamma_{l+1}^{\prime}\right),
$$

where we now view $S_{l+1}$ as lying in $\left(M_{l}, \gamma_{l}\right) \subset\left(M_{l}^{\prime \prime}, \gamma_{l}^{\prime \prime}\right)$. Certainly $B_{l+1}^{\prime}$ satisfies conditions (1) and (2).

Consider condition (3). We begin by considering a component $F$ of $\partial_{h} N\left(B_{l}^{\prime \prime}\right)$. The surface $F$ can be classified as one of the following 3 types (see Figure 3, right):

(1) $F$ can be viewed as a component $G$ of $\partial_{h} N\left(B_{l}^{\prime}\right)$, as illustrated in Figure 3, right. Since the new branch sectors are attached to $B_{l}^{\prime}$ along cusp circles, $L_{F}$ is obtained from $L_{G}$ by adding curves parallel to curves in $L_{G}$ with coherent induced branch direction, where $L_{G}$ is the projection of the branch locus of

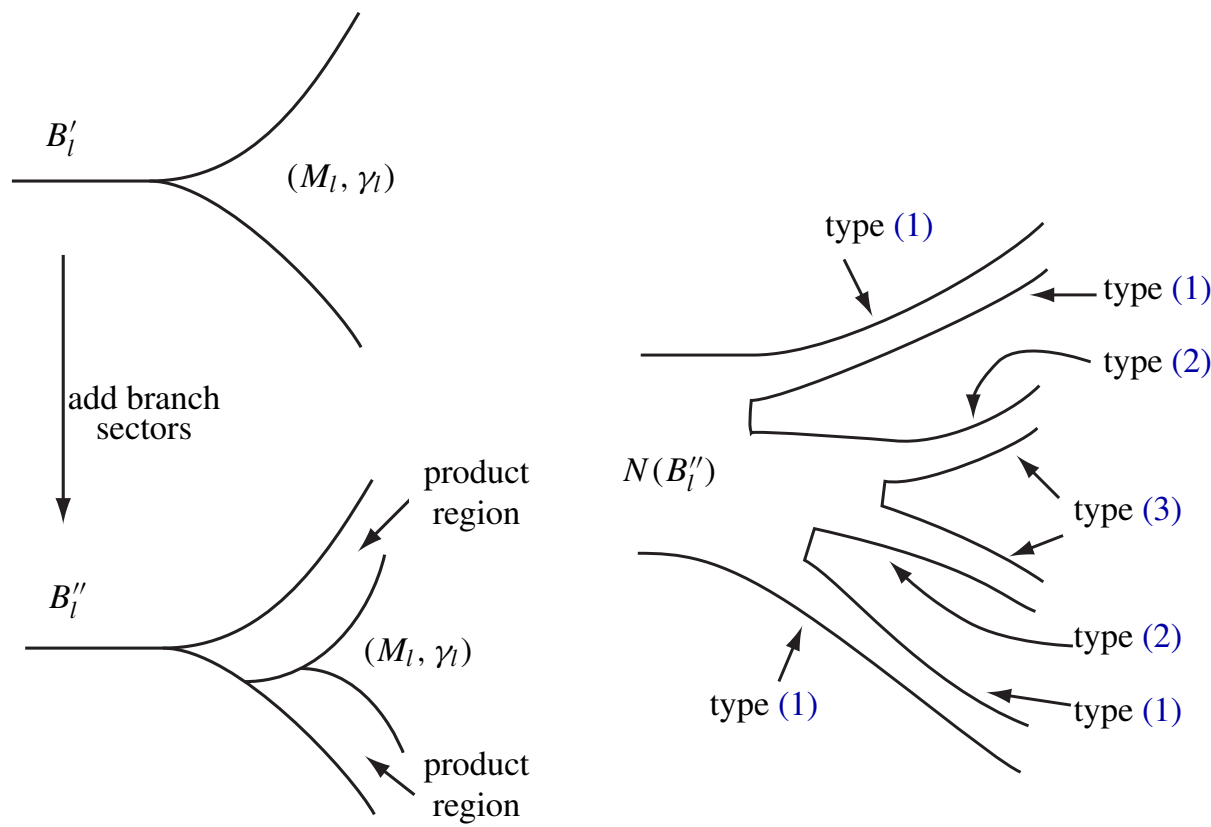

Figure 3. Left: adding branch sectors. Right: three different classifications of a component of $\partial_{h} N\left(B_{l}^{\prime \prime}\right)$. 
$B_{l}^{\prime}$ to $G$. Since the branch directions are coherent, adding such parallel curves to $L_{G}$ does not affect the good arcs in $G$. Thus in this case $F$ is good with respect to $B_{l}^{\prime \prime}$ with the same set of good arcs as $G$.

(2) $F$ is a horizontal boundary component for a newly created product complementary region and $\pi(F)$ contains part of the branch sectors added to $B_{l}^{\prime}$, as illustrated in Figure 3, right. In this case, each component of $L_{F}$ consists of a circle $C$ parallel to the internal boundary and with induced branch direction pointing to the internal boundary and possibly a collection of essential arcs in the annulus between $C$ and the internal boundary.

(3) $F$ is in the boundary of the sutured submanifold $\left(M_{l}, \gamma_{l}\right) \subset\left(M_{l}^{\prime \prime}, \gamma_{l}^{\prime \prime}\right)$. In this case, $L_{F}=\varnothing$.

Next consider how $\partial_{h} N\left(B_{l+1}^{\prime}\right)$ is related to $\partial_{h} N\left(B_{l}^{\prime \prime}\right)$. Let $H$ be a component of $\partial_{h} N\left(B_{l+1}^{\prime}\right)$. Then either $H$ can be viewed as a component of $\partial_{h} N\left(B_{l}^{\prime \prime}\right)$ or $H$ contains a subset of one side of $S_{l+1}$. Our goal is to find a set of good arcs for each component $H$ of $\partial_{h} N\left(B_{l+1}^{\prime}\right)$, so that the projections of the good arcs in $B_{l+1}^{\prime}$ are disjoint.

Case (a). $H$ is not a component of $\partial_{h} N\left(B_{l}^{\prime \prime}\right)$

In this case, $H$ is contained in the union of one side of $S_{l+1}$ and $F \backslash \partial S_{l+1}$, where $F$ is a component of $\partial_{h} N\left(B_{l}^{\prime \prime}\right)$ of type (3). By our construction, $L_{F}=\varnothing$. Moreover, on the other side of $F$, there is a corresponding component $F^{\prime}$ of $\partial_{h} N\left(B_{l}^{\prime \prime}\right)$ of type (2) such that $\pi(F) \cap \pi\left(F^{\prime}\right) \neq \varnothing$ in the branched surface $B_{l}^{\prime \prime}$. Adding $S_{l+1}$ to $B_{l}^{\prime \prime}$ does not affect $F^{\prime}$, so we may also view $F^{\prime}$ as a component of $\partial_{h} N\left(B_{l+1}^{\prime}\right)$. Next we choose good arcs for both $H$ and $F^{\prime}$.

First note that since the original sutured manifold decomposition is well-groomed, $\partial S_{l+1}$ is homologically nontrivial in $H_{1}(F, \partial F)$. There is a simple closed curve $\eta$ in $F$ transverse to $S_{l+1}$, as shown in Figure 4 (note that the arrows in Figure 4 on $\partial S_{l+1}$ denote the branch direction at $\partial S_{l+1}$ ), such that the algebraic intersection

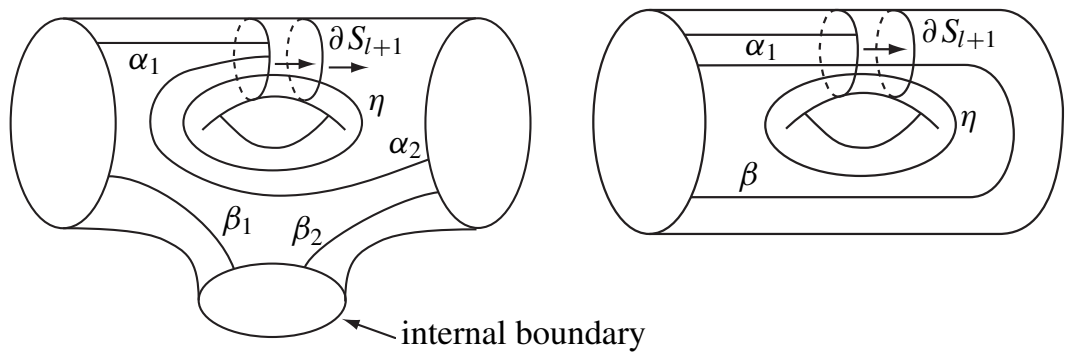

Figure 4. Left: arcs connecting each component of $\partial F \cap M$ to a component of $\partial S_{l+1}$. Right: arcs connecting each component of $\partial F^{\prime} \cap \partial M$ to the internal boundary of $F^{\prime}$. 
number of $\eta$ and $\partial S_{l+1}$ is equal to $\left|\eta \cap \partial S_{l+1}\right|$ (this is equivalent to saying that the normal direction of $\partial S_{l+1}$ at $\eta \cap \partial S_{l+1}$, induced from the branch direction of $B_{l+1}^{\prime}$, are coherent along $\eta$ ).

Recall that $H$ can be viewed as the union of one side of $S_{l+1}$ and $F \backslash \partial S_{l+1}$. We first consider the components $\theta_{1}, \ldots, \theta_{p}$ of $\partial H \cap \partial M$ that are not in $F$ (i.e., each $\theta_{i}$ can be viewed as a component of $\left.\partial S_{l+1} \cap \partial M\right)$. We can find an arc $\gamma_{i}$ connecting $\theta_{i}$ to the internal boundary of $H$ such that $\gamma_{i}$ either is totally in (one side of) $S_{l+1}$ or consists an arc in $S_{l+1}$ and an arc in $F$ parallel to a subarc of $\eta$. Moreover, we can choose these arcs $\gamma_{i}$ to be disjoint in $H$.

Now we consider the components of $\partial F \cap \partial M$ (which are viewed as components of $\partial H \cap \partial M)$. It is easy to see from our construction that there is a collection of disjoint good $\operatorname{arcs} \alpha_{1}, \ldots, \alpha_{q}$ in $F$ (see the $\operatorname{arcs} \alpha_{1}$ and $\alpha_{2}$ in Figure 4, left), such that (1) these arcs $\alpha_{j}$ connect each component of $\partial F \cap \partial M$ to a component of $\partial S_{l+1}$, and (2) these arcs $\alpha_{j}$ are disjoint from the curve $\eta$ describe above.

It follows from our construction that these arcs $\gamma_{i}$ and $\alpha_{j}$ form a set of good arcs $\Gamma_{H}$ for $H$.

Next we consider the component $F^{\prime}$ of $\partial_{h} N\left(B_{l}^{\prime \prime}\right)$ on the other side of $F . F^{\prime}$ is a type (2) component of $\partial_{h} N\left(B_{l}^{\prime \prime}\right)$, and we may view $F^{\prime}$ as a component of $\partial_{h} N\left(B_{l+1}^{\prime}\right)$. Moreover, we view $F^{\prime}$ as a parallel copy of $F$ and view the curves $\partial S_{l+1}, \eta$ and $\alpha_{j}$ described above as curves in $F^{\prime}$. We have two slightly different situations. The first is that $F^{\prime}$ (and hence $F$ ) has nonempty internal boundary, and the second is that $F^{\prime}$ has no internal boundary.

If $F^{\prime}$ has nonempty internal boundary, then there are $\operatorname{arcs} \beta_{1}, \ldots, \beta_{r}$ in $F^{\prime}$ (see the $\operatorname{arcs} \beta_{1}$ and $\beta_{2}$ in Figure 4, left), such that (1) the arcs $\beta_{k}$ connect each component of $\partial F^{\prime} \cap \partial M$ to the internal boundary of $F^{\prime}$, and (2) the arcs $\beta_{k}$ are disjoint from $\eta$, $\partial S_{l+1}$ and the $\operatorname{arcs} \alpha_{j}$. The $\operatorname{arcs} \beta_{k}$ form a set of $\operatorname{good} \operatorname{arcs} \Gamma_{F^{\prime}}$ for $F^{\prime}$. Moreover, since each $\beta_{k}$ is disjoint from $\eta$ and the $\operatorname{arcs} \alpha_{j}$, the projections $\pi\left(\Gamma_{H}\right)$ and $\pi\left(\Gamma_{F^{\prime}}\right)$ of the good arcs $\Gamma_{H}$ and $\Gamma_{F^{\prime}}$ for $H$ and $F^{\prime}$ respectively are disjoint in $B_{l+1}^{\prime}$.

If $F^{\prime}$ does not have internal boundary (in which case $F^{\prime}$ must be a Seifert surface of the knot exterior), then as shown in Figure 4, right, there is an $\operatorname{arc} \beta$ properly embedded in $F^{\prime}$ such that (1) $\beta$ is disjoint from $\eta$ and the arcs $\alpha_{j}$ and (2) the intersection of $\beta$ with $\partial S_{l+1}$ is minimal up to isotopy. Since the original sutured manifold is well-groomed, the requirement (2) implies that the algebraic intersection number of $\beta$ and $\partial S_{l+1}$ is equal to $\left|\beta \cap \partial S_{l+1}\right|$. Thus $\beta$ is a good arc for $F^{\prime}$. Since $\beta$ is chosen to be disjoint from $\eta$ and each $\alpha_{j}$, the projections of $\pi(\beta)$ and $\pi\left(\Gamma_{H}\right)$ on $B_{l+1}^{\prime}$ are disjoint.

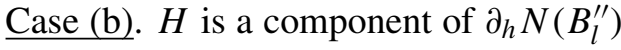

In this case, either $L_{H}$ is unchanged by the decomposition by $S_{l+1}$ or $H$ is the surface $F^{\prime}$ of type (2) considered in Case (a). In Case (a), we have already 
constructed a set of good arcs for the type (2) surface $F^{\prime}$, so we may assume that $L_{H}$ is unchanged by the decomposition by $S_{l+1}$. Since $H$ (viewed as a component of $\left.\partial_{h} N\left(B_{l}^{\prime \prime}\right)\right)$ is good in $B_{l}^{\prime \prime}, H$ is good in $B_{l+1}^{\prime}$. Furthermore, the projections of the good arcs in Case (a) and the good arcs (from the induction) of $H$ in this case are disjoint in $B_{l+1}^{\prime}$.

So $B_{l+1}^{\prime}$ is good. It remains to show that $B_{l+1}^{\prime}$ does not carry any torus. Since $B_{l}^{\prime}$ does not carry any torus and $B_{l}^{\prime \prime}$ can be obtained by splitting $B_{l}^{\prime}, B_{l}^{\prime \prime}$ does not carry any torus. Suppose $B_{l+1}^{\prime}$ carries a torus $T$. Then $T$ can be expressed as the union of some copies of $S_{l+1}$ and a surface in $N\left(B_{l}^{\prime \prime}\right)$ transverse to the $I$-fibers. Moreover, the transverse orientation of the branched surface induces a compatible normal orientation for $T$. Since the original sutured manifold decomposition sequence is well-groomed, $\partial S_{l+1} \cap R_{ \pm}\left(\gamma_{l}\right)$ is a collection of homologically nontrivial curves in $H_{1}\left(R_{ \pm}\left(\gamma_{l}\right), \partial R_{ \pm}\left(\gamma_{l}\right)\right)$. Thus there is a component $F$ of $\partial_{h} N\left(B_{l}^{\prime \prime}\right)$, such that $T \cap F$ (with the induced orientation) is homologically nontrivial in $F$. However, since $T$ is a torus in $S^{3}, T$ is homologically trivial and this is impossible.

Therefore, $B_{l+1}^{\prime}$ satisfies properties (1)-(4) of the lemma and we can inductively construct the sutured manifold hierarchy and corresponding sequence of branched surfaces as claimed.

Step 2: Adding product disks. Let $B_{n}^{\prime}$ be the good branched surface constructed in the proof of Lemma 4.4. It follows from the conditions on the sutured manifold hierarchy and our construction above that $\partial B_{n}^{\prime}$ consists of circles of slope 0 in the torus $\partial M$. In this section, we will add some product disks and modify $B_{n}^{\prime}$ to get a laminar branched surface carrying more laminations.

As $M \backslash \operatorname{int}\left(N\left(B_{n}^{\prime}\right)\right)$ is a product, we may suppose $M \backslash \operatorname{int}\left(N\left(B_{n}^{\prime}\right)\right)=S \times I$, where $S$ is a compact and possibly disconnected surface. Let $S_{+}=S \times\{0\}$ and $S_{-}=S \times\{1\}$. So $\partial_{h} N\left(B_{n}^{\prime}\right)=S_{+} \cup S_{-}$. It is possible to decompose $S \times I$ as the disjoint union

$$
S \times I=(F \times I) \cup(G \times I),
$$

where $F$ is the union of the components of $S$ without internal boundary. Thus $\partial F \subset \partial M$ and each component of $G$ has nonempty internal boundary. Moreover, each component of $F$ must be a Seifert surface in the knot exterior. Note that, since we take parallel copies of surfaces in the horizontal boundary in each step of the sutured manifold decompositions (see Lemma 4.4), $F \neq \varnothing$. Furthermore, $G=\varnothing$ only if $k$ is fibered.

Let $m=\left|\partial S_{ \pm} \cap \partial M\right|$ be the number of components of the noninternal boundary $S_{ \pm} \cap \partial M$. Since $B_{n}^{\prime}$ is good, there is a collection of pairwise disjoint good arcs in $S_{+}$, denoted by $\eta_{1}, \ldots, \eta_{m}$, and a collection of pairwise disjoint good arcs in $S_{-}$, denoted by $\delta_{1}, \ldots, \delta_{m}$, such that $\pi\left(\bigcup_{i} \eta_{i}\right) \cap \pi\left(\bigcup_{i} \delta_{i}\right)=\varnothing$ (in $\left.B_{n}^{\prime}\right)$ and each component of $\partial S_{ \pm} \cap \partial M$ has exactly one incident good arc $\eta_{i}$ and one incident 
good $\operatorname{arc} \delta_{i}$ attached to it. After relabeling as necessary, we may assume that for $1 \leq i \leq r, \eta_{i}$ and $\delta_{i}$ lie in $F \times\{0,1\}$, while for $r+1 \leq i \leq m, \eta_{i}$ and $\delta_{i}$ lie in $G \times\{0,1\}$. It follows that each $\eta_{i}$ and each $\delta_{i}, 1 \leq i \leq r$, has both endpoints lying on $\partial M$ while each $\eta_{i}$ and $\delta_{i}, r+1 \leq i \leq m$, has exactly one endpoint lying on $\partial M$.

Consider first $F \times[0,1]$. Recall that each component of $F$ is a Seifert surface of the knot exterior. Let $F_{1}$ be any component of $F$ and relabel as necessary so that $\eta_{1} \subset F_{1} \times\{0\}$ and $\delta_{1} \subset F_{1} \times\{1\}$. By [Roberts 2001a, Lemma 4.4], there is a sequence of simple arcs

$$
\alpha_{0}=\eta_{1}, \alpha_{1}, \ldots, \alpha_{l}=\delta_{1}
$$

such that $\alpha_{i} \cap \alpha_{i+1}=\varnothing$ and a regular neighborhood of $\alpha_{i} \cup \alpha_{i+1} \cup \partial F_{1}$ in $F_{1}$ is a twice-punctured torus for each $i, 1 \leq i \leq l$. For $1 \leq i \leq l$, let $F_{1}$ induce a consistent orientation on each $F_{1} \times\left\{\frac{i}{l+1}\right\}$ and orient the disks $\alpha_{i} \times\left[\frac{i}{l+1}, \frac{i+1}{l+1}\right]$ arbitrarily. Add branch sectors to $B_{n}^{\prime}$ as prescribed by the following sequence of sutured manifold decompositions:

$$
\left(M_{n}^{\prime}, \gamma_{n}^{\prime}\right) \stackrel{A}{\rightsquigarrow}\left(M_{n+1}^{\prime}, \gamma_{n+1}^{\prime}\right) \stackrel{B}{\rightsquigarrow}\left(M_{F_{1}}, \gamma_{F_{1}}\right),
$$

where

$$
A=F_{1} \times\left\{\frac{1}{l+1}, \ldots, \frac{l}{l+1}\right\} \quad \text { and } \quad B=\bigcup_{i}\left(\alpha_{i} \times\left[\frac{i}{l+1}, \frac{i+1}{l+1}\right]\right) .
$$

Repeat for each remaining component of $F$ and let $\left(M_{F}, \gamma_{F}\right)$ denote the resulting sutured manifold. Set $B_{F}=B_{\left(M_{F}, \gamma_{F}\right)}$. Notice that the conditions satisfied by the $\operatorname{arcs} \alpha_{i}$ guarantee that $B_{F}$ is laminar.

Now consider $G \times I$. Let $G_{1}$ be a component of $G$ and let $p=\left|\partial G_{1} \cap \partial M\right|$. Let $\left\{C_{1}, \ldots, C_{p}\right\}$ be a listing of the components of $G_{1} \cap \partial M$. After relabeling as necessary, we may assume $\eta_{r+1}, \ldots, \eta_{r+p}$ lie in $G_{1} \times\{0\}$ and $\delta_{r+1}, \ldots, \delta_{r+p}$ lie in $G_{1} \times\{1\}$, with $\left\{\eta_{r+i}(0), \delta_{r+i}(0)\right\} \subset C_{i}$ for each $1 \leq i \leq p$.

Lemma 4.5. Let $\left\{\alpha_{1}, \ldots, \alpha_{p}\right\}$ and $\left\{\beta_{1}, \ldots, \beta_{p}\right\}$ each be a set of pairwise disjoint arcs properly embedded in $G_{1}$ with $\left\{\alpha_{i}(0), \beta_{i}(0)\right\} \subset C_{i}$ and $\left\{\alpha_{i}(1), \beta_{i}(1)\right\} \subset$ $\partial G \backslash\left\{C_{1}, \ldots, C_{p}\right\}$, the internal boundary of $G_{1}$. Let $s=\left|\bigcup_{i} \alpha_{i} \cap \bigcup_{i} \beta_{i}\right|$. Then either $s=0$ or there is a set $\left\{\gamma_{1}, \ldots, \gamma_{p}\right\}$ of pairwise disjoint arcs properly embedded in $G_{1}$ with $\gamma_{i}(0) \in C_{i}, \gamma_{i}(1) \in \partial G_{1} \backslash\left\{C_{1}, \ldots, C_{p}\right\}$, such that

$$
\max \left\{\left|\bigcup_{i} \alpha_{i} \cap \bigcup_{i} \gamma_{i}\right|,\left|\bigcup_{i} \beta_{i} \cap \bigcup_{i} \gamma_{i}\right|\right\}<s .
$$

Proof. Suppose $s \neq 0$. Relabeling as necessary, we may assume that $\alpha_{1}$ and $\bigcup_{i} \beta_{i}$ intersect. Choose $z$ to be the point in $\alpha_{1} \cap \bigcup_{i} \beta_{i}$ that is furthest along $\alpha_{1}$. So there are $j, t_{0}, t_{1}$ such that $z=\alpha_{1}\left(t_{0}\right)=\beta_{j}\left(t_{1}\right)$ and $\alpha_{1}\left(t_{0}, 1\right] \cap \bigcup_{i} \beta_{i}=\varnothing$. Let $\gamma_{j}$ be the concatenation of the two $\operatorname{arcs} \beta_{j}\left[0, t_{1}\right]$ and $\alpha_{1}\left[t_{0}, 1\right]$, perturbed slightly so that it intersects $\alpha_{1}$ transversely and minimally. For $i \neq j$, set $\gamma_{i}=\beta_{i}$. Then $\left|\bigcup_{i} \alpha_{i} \cap \bigcup_{i} \gamma_{i}\right|<\left|\bigcup_{i} \alpha_{i} \cap \bigcup_{i} \beta_{i}\right|$ and $\left|\bigcup_{i} \gamma_{i} \cap \bigcup_{i} \beta_{i}\right|=0$. 
The next corollary follows immediately.

Corollary 4.6. There are sets of arcs $\mathscr{A}_{i}=\left\{\alpha_{1}^{i}, \ldots, \alpha_{p}^{i}\right\}, 1 \leq i \leq q$, such that

(1) for each $i$, the arcs in $\mathscr{A}_{i}$ are pairwise disjoint and properly embedded in $G_{1}$, $\alpha_{j}^{i}(0) \in C_{j}$, and $\alpha_{j}^{i}(1) \in \partial G_{1} \backslash\left\{C_{1}, \ldots, C_{p}\right\}, j=1, \ldots, p$,

(2) $\mathscr{A}_{0}=\left\{\eta_{r+1}, \ldots, \eta_{r+p}\right\}$ and $\mathscr{A}_{q+1}=\left\{\delta_{r+1}, \ldots, \delta_{r+p}\right\}$, and

(3) $\bigcup_{j} \alpha_{j}^{i} \cap \bigcup_{j} \alpha_{j}^{i+1}=\varnothing$ for each $i$.

For $1 \leq i \leq q$, let $G_{1}$ induce a consistent orientation on each $G_{1} \times\left\{\frac{i}{q+1}\right\}$. Orient the disks $\alpha_{j}^{i} \times\left[\frac{i}{q+1}, \frac{i+1}{q+1}\right]$ so that the orientation induced on their boundaries agrees with the orientation of $\alpha_{j}^{i}$ (which is the orientation from its starting point in $\partial M$ to its ending point in the internal boundary). Add branch sectors to $B_{F}$ as given by the following sequence of sutured manifold decompositions:

$$
\left(M_{F}, \gamma_{F}\right) \stackrel{A}{\rightsquigarrow}\left(M_{F}^{\prime}, \gamma_{F}^{\prime}\right) \stackrel{B}{\rightsquigarrow}\left(M_{G_{1}}, \gamma_{G_{1}}\right),
$$

where

$$
A=G_{1} \times\left\{\frac{1}{q+1}, \ldots, \frac{q}{q+1}\right\} \quad \text { and } \quad B=\bigcup_{i, j}\left(\alpha_{j}^{i} \times\left[\frac{i}{q+1}, \frac{i+1}{q+1}\right]\right) .
$$

Repeat for each remaining component of $G$ and let $\left(M_{G}, \gamma_{G}\right)$ denote the resulting sutured manifold. Set $B_{G}=B_{\left(M_{G}, \gamma_{G}\right)}$. Notice that the conditions satisfied by the $\operatorname{arcs} \alpha_{j}^{i}$ guarantee that $B_{G}$ is laminar.

By Lemma $4.4, B_{n}^{\prime}$ does not carry any torus. Therefore, any branched surface obtained by splitting $B_{n}^{\prime}$ also cannot carry a torus. And finally, any (closed) torus carried by $B_{G}$ but not by this splitting of $B_{n}^{\prime}$ would necessarily pass through one of the added disk branches and hence would necessarily have nonempty boundary. Thus $B_{G}$ does not carry a torus.

Noting that for each product disk in the above construction, its two normal directions give two ways of deforming it into a branched surface, let $B_{G}^{\prime}$ denote the branched surface obtained from $B_{G}$ by reversing the orientations of the disks $\alpha_{j}^{i} \times\left[\frac{i}{q+1}, \frac{i+1}{q+1}\right]$. Notice that $B_{G}^{\prime}$ is also laminar, has only product complementary regions, and does not carry a torus.

Hence we have laminar branched surfaces $B_{G}$ and $B_{G}^{\prime}$ with only product complementary regions and which do not carry a torus. We may therefore apply Theorem 2.2 to conclude the existence of taut foliations realizing any boundary slope carried by $B_{G} \cap \partial M$ or $B_{G}^{\prime} \cap \partial M$. It remains to compute these boundary slopes.

The boundary train tracks. Let $\tau$ denote the train track $B_{G} \cap \partial M$ and let $\tau^{\prime}$ denote the train track $B_{G}^{\prime} \cap \partial M$.

Lemma 4.7. Together, $\tau$ and $\tau^{\prime}$ realize all slopes in $(-a, b)$ for some $a, b>0$. 


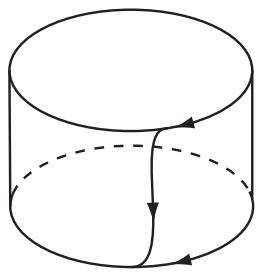

(a)

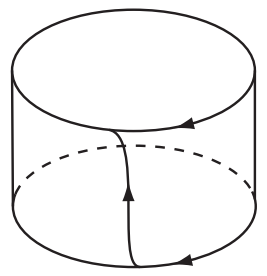

(b)

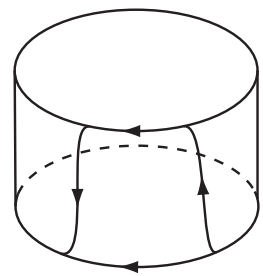

(c)

Figure 5. Train tracks that realize all slopes in $(-a, b)$ for $a, b>0$.

Proof. Consider an annular component $A_{G}$ of $\partial G_{1} \times\left[\frac{i}{q+1}, \frac{i+1}{q+1}\right]$. The train tracks $\tau$ and $\tau^{\prime}$ restricted to $A_{G}$ have the form indicated in parts (a) and (b), respectively, of Figure 5. Similarly, consider an annular component $A_{F}$ of $\partial F_{1} \times\left[\frac{i}{l+1}, \frac{i+1}{l+1}\right]$. Recall that each $F_{1} \times\left\{\frac{i}{l+1}\right\}$ is a Seifert surface and the good arc for $F_{1}$ has both endpoints on the circle $\partial F_{1}$. Thus both $\tau$ and $\tau^{\prime}$ restricted to $A_{F}$ have the form indicated in Figure 5(c). Call all such nonlongitudinal branches of $\tau$ or $\tau^{\prime}$ vertical.

Since all vertical branches of $\tau$ (or $\tau^{\prime}$, respectively) are of one of the three types shown in Figure 5, it follows that $\tau$ (or $\tau^{\prime}$ ) is a train track obtained by concatenating pieces of the types of Figure 5(a) or (c) (or (b) or (c), respectively). Examples are shown in Figure 6. Notice that $\tau$ and $\tau^{\prime}$ are orientable and measurable; namely, they admit a transverse measure [Hatcher 1988, page 66; Penner and Harer 1992, page 86]. Assign weights $x, y$, and $x+y$ to the vertical branches of $\tau$ and $\tau^{\prime}$ as indicated in Figure 6; namely, vertical branches in $G \times I$ regions are weighted $x$, the compatibly oriented branches in $F \times I$ regions are weighted $x+y$, and the remaining branches in $F \times I$ regions are weighted $y$. Then assign weights from $\{1,1+x, 1+y, 1+x+y\}$ to the remaining branches of $\tau$ and $\tau^{\prime}$ to obtain a measure $\mu$ on $\tau$ and a measure $\mu^{\prime}$ on $\tau^{\prime}$.
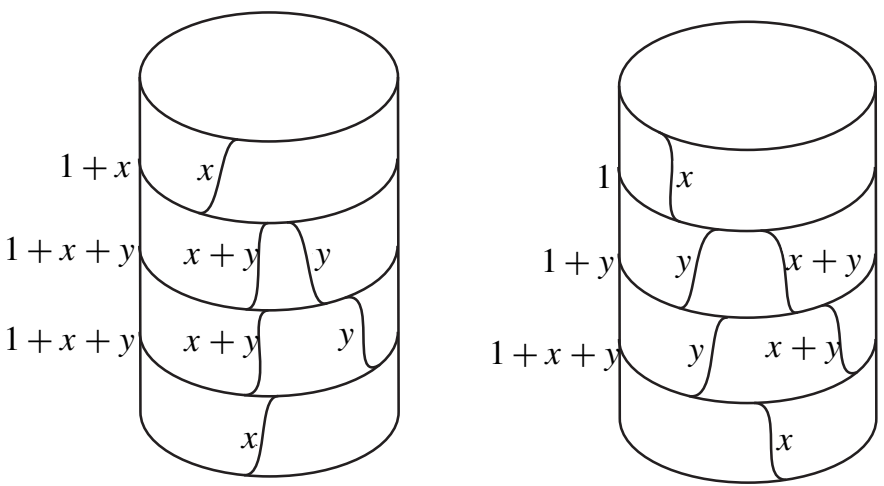

Figure 6. Examples of train tracks. 
Recall that if $\gamma$ is a simple closed curve in a torus, then the slope of $\gamma$ is given in standard coordinates by

$$
\operatorname{slope}(\gamma)=\frac{\langle\lambda, \gamma\rangle}{\langle\gamma, m\rangle}
$$

where $\langle$,$\rangle denotes algebraic intersection number and \lambda$ is the longitude and $m$ is the meridian of the knot $k$ in $S^{3}$.

Applying (1) to the measured train tracks $(\tau, \mu)$ and $\left(\tau^{\prime}, \mu^{\prime}\right)$ while letting $x, y$ range over all values $0<y \ll x$, we see that $(\tau, \mu)$ and $\left(\tau^{\prime}, \mu^{\prime}\right)$ together carry all boundary slopes in some open interval $(-a, b)$ about 0 .

By Theorem 2.2, if $\tau$ (or $\tau^{\prime}$ ) fully carries a curve of slope $s$, then $B_{G}$ (or $B_{G}^{\prime}$, respectively) fully carries an essential lamination whose boundary consists of loops of slope $s$ in $\partial M$. Moreover, this lamination extends to an essential lamination in $M(s)$. Since $M \backslash \operatorname{int}\left(N\left(B_{G}\right)\right)$ and $M \backslash \operatorname{int}\left(N\left(B_{G}^{\prime}\right)\right)$ consist of product regions, such essential laminations can be extended to taut foliations. This proves Theorem 1.1.

\section{References}

[Altman 2012] I. Altman, "The sutured Floer polytope and taut depth one foliations", preprint, 2012. arXiv 1205.0442

[Boyer et al. 2012] S. Boyer, C. M. Gordon, and L. Watson, "On L-spaces and left-orderable fundamental groups", Math. Ann. 2012 (2012), 1-33.

[Calegari and Dunfield 2003] D. Calegari and N. M. Dunfield, "Laminations and groups of homeomorphisms of the circle", Invent. Math. 152:1 (2003), 149-204. MR 2005a:57013 Zbl 1025.57018

[Candel and Conlon 2003] A. Candel and L. Conlon, Foliations, II, Graduate Studies in Mathematics 60, American Mathematical Society, Providence, RI, 2003. MR 2004e:57034 Zbl 1035.57001

[Cantwell and Conlon 2012] J. Cantwell and L. Conlon, "Smoothability of Gabai's foliations", preprint, 2012.

[Dasbach and Li 2004] O. T. Dasbach and T. Li, "Property P for knots admitting certain Gabai disks", Topology Appl. 142:1-3 (2004), 113-129. MR 2005d:57006 Zbl 1062.57004

[Delman and Roberts 1999] C. Delman and R. Roberts, "Alternating knots satisfy strong property P", Comment. Math. Helv. 74:3 (1999), 376-397. MR 2001g:57009 Zbl 0949.57002

[Eliashberg and Thurston 1998] Y. M. Eliashberg and W. P. Thurston, Confoliations, University Lecture Series 13, American Mathematical Society, Providence, RI, 1998. MR 98m:53042 Zbl 0893.53001

[Gabai 1983] D. Gabai, "Foliations and the topology of 3-manifolds", J. Differential Geom. 18:3 (1983), 445-503. MR 86a:57009 Zbl 0533.57013

[Gabai 1987a] D. Gabai, "Foliations and the topology of 3-manifolds, II", J. Differential Geom. 26:3 (1987), 461-478. MR 89a:57014a Zbl 0627.57012

[Gabai 1987b] D. Gabai, "Foliations and the topology of 3-manifolds, III", J. Differential Geom. 26:3 (1987), 479-536. MR 89a:57014b Zbl 0639.57008 
[Gabai 1991] D. Gabai, "Foliations and 3-manifolds", pp. 609-619 in Proceedings of the International Congress of Mathematicians, I (Kyoto, 1990), edited by I. Satake, Math. Soc. Japan, Tokyo, 1991. MR 93d:57013 Zbl 0754.57008

[Gabai and Oertel 1989] D. Gabai and U. Oertel, "Essential laminations in 3-manifolds", Ann. of Math. (2) 130:1 (1989), 41-73. MR 90h:57012 Zbl 0685.57007

[Ghiggini 2008] P. Ghiggini, "Knot Floer homology detects genus-one fibred knots", Amer. J. Math. 130:5 (2008), 1151-1169. MR 2010f:57013 Zbl 1149.57019

[Haefliger 1962] A. Haefliger, "Variétés feuilletées", Ann. Scuola Norm. Sup. Pisa (3) 16 (1962), 367-397. MR 32 \#6487 Zbl 0122.40702

[Hatcher 1988] A. E. Hatcher, "Measured lamination spaces for surfaces, from the topological viewpoint”, Topology Appl. 30:1 (1988), 63-88. MR 89k:57022 Zbl 0662.57005

[Hedden and Levine 2012] M. Hedden and A. S. Levine, "Splicing knot complements and bordered Floer homology", preprint, 2012. arXiv 1210.7055

[Hedden and Watson 2010] M. Hedden and L. Watson, "Does Khovanov homology detect the unknot?”, Amer. J. Math. 132:5 (2010), 1339-1345. MR 2012c:57023 Zbl 1204.57010

[Juhász 2008] A. Juhász, "Floer homology and surface decompositions", Geom. Topol. 12:1 (2008), 299-350. MR 2009a:57021 Zbl 1167.57005

[Kazez and Roberts 2014] W. H. Kazez and R. Roberts, “Approximating $C^{0}$ taut foliations”, preprint, 2014. arXiv 1404.5919

[Li 2002] T. Li, "Laminar branched surfaces in 3-manifolds", Geom. Topol. 6 (2002), 153-194. MR 2003h:57019 Zbl 1067.57011

[Li 2003] T. Li, "Boundary train tracks of laminar branched surfaces", pp. 269-285 in Topology and geometry of manifolds (Athens, GA, 2001), edited by G. Matić and C. McCrory, Proc. Sympos. Pure Math. 71, Amer. Math. Soc., Providence, RI, 2003. MR 2005h:57028 Zbl 1043.57006

[Novikov 1965] S. P. Novikov, "The topology of foliations”, Trudy Moskov. Mat. Obšč. 14 (1965), 248-278. In Russian; translated in Trans. Moscow Math. Soc. 14 (1965), 268-304. MR 34 \#824 Zbl 0247.57006

[Ozsváth and Szabó 2004a] P. Ozsváth and Z. Szabó, "Heegaard diagrams and holomorphic disks", pp. 301-348 in Different faces of geometry, edited by S. Donaldson et al., Int. Math. Ser. (N. Y.) 3, Kluwer, New York, 2004. MR 2005g:57057 Zbl 1091.57010

[Ozsváth and Szabó 2004b] P. Ozsváth and Z. Szabó, "Holomorphic disks and genus bounds", Geom. Topol. 8 (2004), 311-334. MR 2004m:57024 Zbl 1056.57020

[Ozsváth and Szabó 2004c] P. Ozsváth and Z. Szabó, "Holomorphic disks and topological invariants for closed three-manifolds", Ann. of Math. (2) 159:3 (2004), 1027-1158. MR 2006b:57016 Zbl 1073.57009

[Ozsváth and Szabó 2004d] P. Ozsváth and Z. Szabó, "Holomorphic disks and three-manifold invariants: properties and applications", Ann. of Math. (2) 159:3 (2004), 1159-1245. MR 2006b:57017 Zbl 1081.57013

[Ozsváth and Szabó 2005] P. Ozsváth and Z. Szabó, "On knot Floer homology and lens space surgeries”, Topology 44:6 (2005), 1281-1300. MR 2006f:57034 Zbl 1077.57012

[Palmeira 1978] C. F. B. Palmeira, "Open manifolds foliated by planes", Ann. Math. (2) 107:1 (1978), 109-131. MR 58 \#18490 Zbl 0382.57010

[Penner and Harer 1992] R. C. Penner and J. L. Harer, Combinatorics of train tracks, Annals of Mathematics Studies 125, Princeton University Press, 1992. MR 94b:57018 Zbl 0765.57001 
[Reeb 1952] G. Reeb, Sur certaines propriétés topologiques des variétés feuilletées, Actualités Sci. Ind. 1183, Hermann, Paris, 1952. MR 14,1113a Zbl 0049.12602

[Roberts 1995] R. Roberts, "Constructing taut foliations", Comment. Math. Helv. 70:4 (1995), 516545. MR 96j:57032 Zbl 0855.57009

[Roberts 2001a] R. Roberts, "Taut foliations in punctured surface bundles, I", Proc. London Math. Soc. (3) 82:3 (2001), 747-768. MR 2003a:57040 Zbl 1034.57017

[Roberts 2001b] R. Roberts, "Taut foliations in punctured surface bundles, II", Proc. London Math. Soc. (3) 83:2 (2001), 443-471. MR 2003j:57016 Zbl 1034.57018

[Roberts et al. 2003] R. Roberts, J. Shareshian, and M. Stein, "Infinitely many hyperbolic 3-manifolds which contain no Reebless foliation”, J. Amer. Math. Soc. 16:3 (2003), 639-679. MR 2004e:57023 Zbl 1012.57022

[Rosenberg 1968] H. Rosenberg, "Foliations by planes", Topology 7 (1968), 131-138. MR 37 \#3595 Zbl 0157.30504

[Thurston 1982] W. P. Thurston, "Three-dimensional manifolds, Kleinian groups and hyperbolic geometry”, Bull. Amer. Math. Soc. (N.S.) 6:3 (1982), 357-381. MR 83h:57019 Zbl 0496.57005

[Thurston 1998] W. P. Thurston, "Three-manifolds, foliations and circles, II", unfinished manuscript, 1998.

Received November 13, 2012. Revised January 16, 2013.

TAO LI

DEPARTMENT OF MATHEMATICS

Boston COLLEGE

CARNEY HALL

140 Commonwealth Ave.

Chestnut Hill, MA 02467

UNITED STATES

taoli@bc.edu

RACHEL ROBERTS

DEPARTMENT OF MATHEMATICS

WASHINGTON UNIVERSITY

ST. LOUIS, MO 63130

UNITED STATES

roberts@math.wustl.edu 


\title{
PACIFIC JOURNAL OF MATHEMATICS
}

\author{
msp.org/pjm
}

Founded in 1951 by E. F. Beckenbach (1906-1982) and F. Wolf (1904-1989)

\section{EDITORS}

Don Blasius (Managing Editor)

Department of Mathematics

University of California

Los Angeles, CA 90095-1555

blasius@math.ucla.edu

\author{
Paul Balmer \\ Department of Mathematics \\ University of California \\ Los Angeles, CA 90095-1555 \\ balmer@math.ucla.edu \\ Robert Finn \\ Department of Mathematics \\ Stanford University \\ Stanford, CA 94305-2125 \\ finn@math.stanford.edu \\ Sorin Popa \\ Department of Mathematics \\ University of California \\ Los Angeles, CA 90095-1555 \\ popa@math.ucla.edu
}

\author{
Vyjayanthi Chari \\ Department of Mathematics \\ University of California \\ Riverside, CA 92521-0135 \\ chari@math.ucr.edu \\ Kefeng Liu \\ Department of Mathematics \\ University of California \\ Los Angeles, CA 90095-1555 \\ liu@math.ucla.edu \\ Jie Qing \\ Department of Mathematics \\ University of California \\ Santa Cruz, CA 95064 \\ qing@ cats.ucsc.edu
}

\section{PRODUCTION}

Silvio Levy, Scientific Editor, production@msp.org

\section{SUPPORTING INSTITUTIONS}

ACADEMIA SINICA, TAIPEI

CALIFORNIA INST. OF TECHNOLOGY

INST. DE MATEMÁTICA PURA E APLICADA

KEIO UNIVERSITY

MATH. SCIENCES RESEARCH INSTITUTE

NEW MEXICO STATE UNIV.

OREGON STATE UNIV.

\author{
STANFORD UNIVERSITY \\ UNIV. OF BRITISH COLUMBIA \\ UNIV. OF CALIFORNIA, BERKELEY \\ UNIV. OF CALIFORNIA, DAVIS \\ UNIV. OF CALIFORNIA, LOS ANGELES \\ UNIV. OF CALIFORNIA, RIVERSIDE \\ UNIV. OF CALIFORNIA, SAN DIEGO \\ UNIV. OF CALIF., SANTA BARBARA
}

\author{
Daryl Cooper \\ Department of Mathematics \\ University of California \\ Santa Barbara, CA 93106-3080 \\ cooper@math.ucsb.edu \\ Jiang-Hua Lu \\ Department of Mathematics \\ The University of Hong Kong \\ Pokfulam Rd., Hong Kong \\ jhlu@maths.hku.hk \\ Paul Yang \\ Department of Mathematics \\ Princeton University \\ Princeton NJ 08544-1000 \\ yang@math.princeton.edu
}

These supporting institutions contribute to the cost of publication of this Journal, but they are not owners or publishers and have no responsibility for its contents or policies.

See inside back cover or msp.org/pjm for submission instructions.

The subscription price for 2014 is US $\$ 410 /$ year for the electronic version, and \$535/year for print and electronic.

Subscriptions, requests for back issues and changes of subscribers address should be sent to Pacific Journal of Mathematics, P.O. Box 4163, Berkeley, CA 94704-0163, U.S.A. The Pacific Journal of Mathematics is indexed by Mathematical Reviews, Zentralblatt MATH, PASCAL CNRS Index, Referativnyi Zhurnal, Current Mathematical Publications and Web of Knowledge (Science Citation Index).

The Pacific Journal of Mathematics (ISSN 0030-8730) at the University of California, c/o Department of Mathematics, 798 Evans Hall \#3840, Berkeley, CA 94720-3840, is published twelve times a year. Periodical rate postage paid at Berkeley, CA 94704, and additional mailing offices. POSTMASTER: send address changes to Pacific Journal of Mathematics, P.O. Box 4163, Berkeley, CA 94704-0163.

PJM peer review and production are managed by EditFLOW ${ }^{\circledR}$ from Mathematical Sciences Publishers.

\section{PUBLISHED BY}

\section{mathematical sciences publishers \\ nonprofit scientific publishing}

http://msp.org/

(C) 2014 Mathematical Sciences Publishers 


\section{PACIFIC JOURNAL OF MATHEMATICS}

Volume 269 No. $1 \quad$ May 2014

The asymptotic behavior of Palais-Smale sequences on manifolds with $\quad 1$ boundary

SÉRGIO AlMARAZ

The cup subalgebra of a $\mathrm{II}_{1}$ factor given by a subfactor planar algebra is maximal amenable

ARNAUD BROTHIER

Representation theory of type B and C standard Levi $W$-algebras

JONATHAN BROWN and SIMON M. GOODWIN

New invariants for complex manifolds and rational singularities

RONG DU and YUN GAO

Homogeneity groups of ends of open 3-manifolds

DENNIS J. GARITY and DUŠAN REPOVŠ

On the concircular curvature of a $(\kappa, \mu, \nu)$-manifold

Florence Gouli-Andreou and Evaggelia Moutafi

Genuses of cluster quivers of finite mutation type

FANG LI, JICHUN LIU and YICHAO YANG

Taut foliations in knot complements

TAO LI and RACHEL ROBERTS

On the set of maximal nilpotent supports of supercuspidal representations 169

QIN YUJUN

The natural filtrations of finite-dimensional modular Lie superalgebras of 199 Witt and Hamiltonian type

Keli Zheng, Yongzheng ZHANG and WeI Song

Free Brownian motion and free convolution semigroups: multiplicative 219 case

PING ZHONG 